\title{
Japanese Man with HCV Genotype 4 Infection and Cirrhosis Who Was Successfully Treated by the Combination of Glecaprevir and Pibrentasvir
}

\author{
Mai Totsuka ${ }^{1}$, Masayuki Honda ${ }^{1}$, Tatsuo Kanda ${ }^{1}$, Tomotaka Ishii ${ }^{1}$, Naoki Matsumoto ${ }^{1}$, \\ Yoichiro Yamana ${ }^{1}$, Tomohiro Kaneko ${ }^{1}$, Taku Mizutani ${ }^{1}$, Hiroshi Takahashi ${ }^{1}$, \\ Mariko Kumagawa ${ }^{1}$, Reina Sasaki ${ }^{1}$, Ryota Masuzaki ${ }^{1}$, Shini Kanezawa ${ }^{1}$, Kazushige Nirei ${ }^{1}$, \\ Hiroaki Yamagami ${ }^{1}$, Shunichi Matsuoka ${ }^{1}$, Hiroshi Ohnishi ${ }^{2}$, \\ Hiroaki Okamoto $^{2}$ and Mitsuhiko Moriyama ${ }^{1}$
}

\begin{abstract}
:
A 74-year-old man with a history of transfusion at 35 years old in Egypt was referred to our hospital. He was infected with hepatitis C virus (HCV) genotype 4 (GT4), which is a rare HCV GT in Japan, and was also diagnosed with hepatic compensated cirrhosis. We safely treated the patient for 12 weeks with the combination of glecaprevir and pibrentasvir, and a sustained virologic response (SVR) was achieved. This is the first report of HCV GT4 infection in a treatment-naïve Japanese patient with cirrhosis in whom SVR was achieved with the combination treatment of glecaprevir and pibrentasvir.
\end{abstract}

Key words: cirrhosis, DAA, HCV genotype 4, Japan, transfusion

(Intern Med 60: 2061-2066, 2021)

(DOI: 10.2169/internalmedicine.6728-20)

\section{Introduction}

Hepatitis C virus (HCV) infection induces acute and chronic hepatitis, hepatic cirrhosis, hepatocellular carcinoma (HCC) and other extrahepatic manifestations. HCV infection is still a leading cause of HCC in Japan (1). Although effective direct-acting antivirals (DAAs) against $\mathrm{HCV}$ have been introduced, making it now easier to achieve a sustained virologic response (SVR) in daily clinical practice, several issues need to be addressed, such as the risk of hepatocarcinogenesis after the achievement of SVR and the long-term prognosis $(2,3)$.

At present, eight HCV genotypes (GTs) are known to exist (4-6). In Japan, HCV GT1b, GT2a and GT2b have a prevalence of $70 \%, 20 \%$ and $10 \%$, respectively (7). HCV GT4, which is a major genotype in Egypt, is a rare HCV GT in Japan (8). A previous study demonstrated that $0.4 \%$
(4/899) of patients infected with HCV have HCV GT4 in Aichi Prefecture, Japan, and these 4 patients with HCV GT4 were hemophilic men who had received blood products from foreign countries (8).

In Japan, the HCV nonstructural protein (NS) 5B inhibitor sofosbuvir-based DAA combination, the combination of the HCV NS3/4A inhibitor glecaprevir and HCV NS5A inhibitor pibrentasvir, and the combination of the $\mathrm{HCV}$ NS3/4A inhibitor grazoprevir and HCV NS5A inhibitor elbasvir are available for the treatment of $\mathrm{HCV}$-infected individuals (9-15). The Japanese National Health Insurance system has recommended the combination of sofosbuvir and ribavirin for 24 weeks or the combination of glecaprevir and pibrentasvir for 12 weeks as the treatment for patients infected with HCV GTs other than GT1 or GT2. However, there are no clinical trials analyzing the effects of the combination of glecaprevir and pibrentasvir on patients with HCV GT4 in Japan, so this combination's efficacy in Japa-

${ }^{1}$ Division of Gastroenterology and Hepatology, Department of Medicine, Nihon University School of Medicine, Japan and ${ }^{2}$ Division of Virology, Department of Infection and Immunity, Jichi Medical University School of Medicine, Japan

Received: November 16, 2020; Accepted: December 10, 2020; Advance Publication by J-STAGE: February 1, 2021

Correspondence to Dr. Tatsuo Kanda, kanda.tatsuo@nihon-u.ac.jp 
Table 1. Laboratory Data before Starting the Combination Treatment of Glecaprevir and Pibrentasvir in the Present Case.

\begin{tabular}{|c|c|c|c|c|c|}
\hline Item & Values & Item & Values & Item & Values \\
\hline \multicolumn{2}{|c|}{ Peripheral Blood } & \multicolumn{4}{|c|}{ Biochemistry } \\
\hline WBC & $3,900 / \mu \mathrm{L}$ & AST & $160 \mathrm{IU} / \mathrm{L}$ & $\mathrm{NH}_{3}$ & $29 \mu \mathrm{g} / \mathrm{dL}$ \\
\hline $\mathrm{RBC}$ & $497 \times 10^{4} / \mu \mathrm{L}$ & ALT & $225 \mathrm{IU} / \mathrm{L}$ & CRP & $0.10 \mathrm{mg} / \mathrm{dL}$ \\
\hline Hemoglobin & $15.7 \mathrm{~g} / \mathrm{dL}$ & LDH & $275 \mathrm{IU} / \mathrm{L}$ & AFP & $86.7 \mathrm{ng} / \mathrm{mL}$ \\
\hline Platelets & $12.1 \times 10^{4} / \mu \mathrm{L}$ & ALP & $238 \mathrm{IU} / \mathrm{L}$ & PIVKA-II & $16 \mathrm{mAU} / \mathrm{mL}$ \\
\hline \multicolumn{2}{|c|}{ Coagulation system } & $\gamma$-GTP & $81 \mathrm{IU} / \mathrm{L}$ & Serology & \\
\hline PT & $84 \%$ & T. Bil & $0.53 \mathrm{mg} / \mathrm{dL}$ & HBsAg & Negative \\
\hline \multirow[t]{6}{*}{ INR } & 1.09 & $\mathrm{TP}$ & $7.0 \mathrm{~g} / \mathrm{dL}$ & Anti-HBs & Negative \\
\hline & & Albumin & $3.6 \mathrm{~g} / \mathrm{dL}$ & Anti-HBc & Negative \\
\hline & & BUN & $20.2 \mathrm{mg} / \mathrm{dL}$ & Anti-HCV & Positive \\
\hline & & Creatinine & $0.70 \mathrm{mg} / \mathrm{dL}$ & HCV RNA & 5.4 LIU/mL \\
\hline & & Glucose & $214 \mathrm{mg} / \mathrm{dL}$ & HCV GT & $4 a$ \\
\hline & & $\mathrm{HbA1c}$ & $7.3 \%$ & Anti-HIV & Negative \\
\hline
\end{tabular}

WBC: white blood cells, RBC: red blood cells, PT: prothrombin time, INR: international normalized ratio, AST: aspartate aminotransferase, ALT: alanine aminotransferase, LDH: lactate dehydrogenase, ALP: alkaline phosphatase, $\gamma$-GTP: $\gamma$-glutamyl transpeptidase, T. Bil: total bilirubin, TP: total protein, BUN: blood urea nitrogen, HbA1c: hemoglobin A1c, $\mathrm{NH}_{3}$ : ammonia, CRP: C-reactive protein, AFP: alpha fetoprotein, PIVKA-II: protein induced by vitamin $\mathrm{K}$ antagonist-II, HBsAg: hepatitis B surface antigen, Anti-HBs: anti-hepatitis B surface antibody, Anti-HBc: anti-hepatitis B core antibody, Anti-HCV: anti-hepatitis C virus antibody, GT: genotype, Anti-HIV: anti-human immunodeficiency virus antibody

nese patients with HCV GT4 is unclear (14). However, of note, it was reported that SVR rates were more than $90-95 \%$ in HCV GT4-infected Egyptian patients treated with combinations of DAAs (16).

We herein report the interferon-free, 12-week combination treatment with glecaprevir and pibrentasvir that successfully led to an SVR in a treatment-naïve Japanese patient with HCV GT4 infection and hepatic cirrhosis.

\section{Case Report}

A 74-year-old man was referred to our hospital because of his positivity for HCV RNA and undetermined HCV genotype to receive treatment to eradicate the virus. In the outpatient clinic, he had shown no symptoms. He had a history of transfusion for typhoid fever at 35 years old in Egypt. He had been diagnosed with positivity for anti-HCV antibody 10 years ago at a clinic near his house in Japan, but he did not receive any antiviral treatment, including interferon or DAAs. He had been receiving irbesartan (100 mg daily) and amlodipine besilate (10 mg daily) for his hypertension and metformin hydrochloride (500 mg daily) for type 2 diabetes mellitus but had no history of surgery. He also had no history of tattooing, drug abuse, or drug allergy. He was a social drinker, and his family had no history of liver disease.

A physical examination showed no signs of ascites, lower leg edema, or disturbance of consciousness. The cirrhotic liver was slightly palpable at his right hypochondrium. Laboratory data before treatment are shown in Table 1. Reduced platelet counts and elevated transaminase levels were observed. His Child-Pugh classification was Grade A (score
5). Although the alpha-fetoprotein level was elevated, no space occupying lesions were detected in the cirrhotic liver by ultrasound or contrast-enhanced computed tomography (Fig. 1). Hepatic ultrasound elastography showed values of $34.3 \mathrm{kPa}$ and $26.1 \mathrm{kPa}$ on a FibroScan 502 with an M probe (Echosens, Paris, France) and shear wave measurement (ARRIETTA 850; Hitachi Medical Systems, Tokyo Japan), respectively, and these values were compatible with hepatic cirrhosis. Upper gastrointestinal endoscopy demonstrated a solitary varix of the esophagus and no varices of the stomach (Fig. 2). We diagnosed him with compensated cirrhosis due to HCV infection without a liver biopsy.

HCV RNA was extracted from his sera before treatment, and nested reverse transcription polymerase chain reaction (RT-PCR) and direct sequencing were performed. Using the HCV-5'-untranslated region-core region (655 nt.) and HCV NS5B region (502 nt.), HCV GT4a infection was confirmed by a phylogenetic tree analysis with the neighbor-joining method (Fig. 3). The primers in the present study were used as previously described (17).

As recommended by the Japanese National Health Insurance system, he received the 12-week oral combination treatment of glecaprevir (300 mg daily) and pibrentasvir (120 mg daily), without any evident adverse events. HCV RNA was not detected at the end of treatment or at 24 weeks after the end of treatment, indicating that he had achieved an SVR at week 24 (Fig. 4). Furthermore, the serum albumin level had also improved to $4.3 \mathrm{~g} / \mathrm{dL}$.

\section{Discussion}

We herein report a treatment-naïve Japanese man with 
(a)

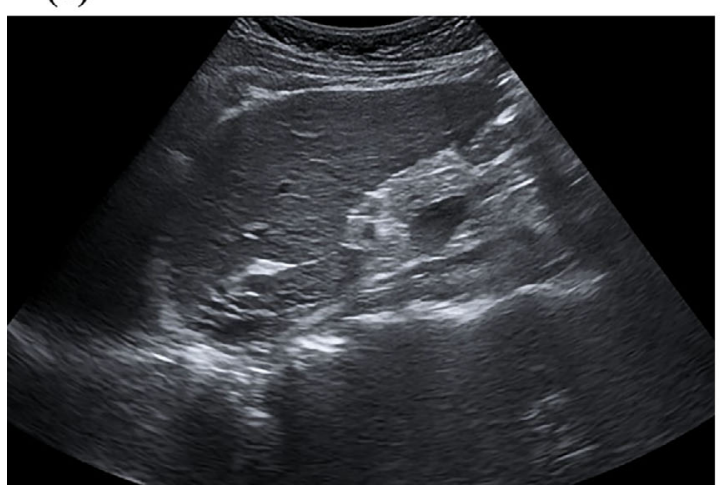

(b)

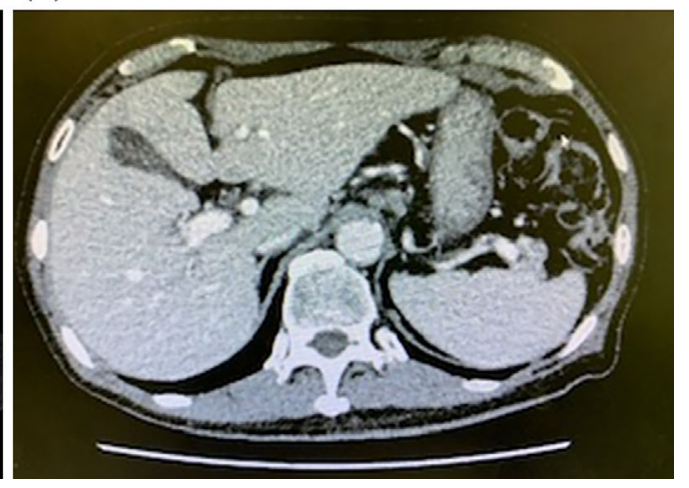

Figure 1. Findings of abdominal ultrasound (US) (a) and computed tomography (CT) (b). (a) US showed a cirrhotic liver with coarse parenchymal pattern, irregular surface, and dull edge but no space-occupying lesions. (b) Contrast-enhanced CT in the portal-dominant phase showed an irregular surface of the liver and splenomegaly with mild dilatation of the paraumbilical vein but no ascites.

(a)



(b)



Figure 2. Findings of upper gastrointestinal endoscopy. (a) Solitary varix of the esophagus. (b) No varices of the stomach.

HCV GT4a infection and hepatic cirrhosis who successfully achieved an SVR with the 12-week combination treatment of glecaprevir and pibrentasvir. The present report is likely to be the first report of the 12 -week combination treatment of glecaprevir and pibrentasvir being effective for a Japanese patient with HCV GT4a infection and hepatic cirrhosis.

It was recently reported that eight HCV GTs exist (4-6). In Egypt, the prevalence of anti-HCV positive rates is relatively high (14.7\% in a 2008 nationwide survey) (18), and anti-schistosomal parenteral therapy and blood transfusion are risk factors for $\mathrm{HCV}$ infection (7). In Egypt, HCV GT4a, GT4b, GT1, and GT3 have a prevalence of 63\%, $30 \%, 6 \%$, and $1 \%$, respectively (7). The present patient had a history of transfusion in Egypt before 1989, when HCV was discovered by molecular biological methods $(19,20)$. As HCV GT4a infection is rare in Japan (8) and hemophilia does not run in his family, the route of infection for $\mathrm{HCV}$ GT4a in this patient was deemed likely to be the transfusion he had undergone in Egypt.
The combination of glecaprevir and pibrentasvir is a pangenotypic DAA therapy for HCV infection. In Japanese clinical trials, 12-week combination treatment of glecaprevir and pibrentasvir for patients with HCV GT1b and GT2 with compensated cirrhosis showed an SVR at week 12 of $100 \%$ $(38 / 38)$ and $100 \%$ (18/18), respectively $(12,13)$. Forns et al. reported an SVR at week 12 of $100 \%(16 / 16)$ in the phase 3 study of the 12-week combination treatment of glecaprevir and pibrentasvir for HCV GT4 patients with compensated cirrhosis (EXPEDITION-1) (21).

In general, the SVR rates in HCV GT4-infected patients treated with the combination of glecaprevir and pibrentasvir are reported to range from $95.5-100 \%$ (Table 2) (22-32). In Japan, clinical trials of this combination treatment for $\mathrm{HCV}$ GT4 have not been performed. The 8-week combination treatment of glecaprevir and pibrentasvir for HCV GT4 treatment-naïve patients with compensated cirrhosis led to $100 \%$ SVR rates $(24,25)$, although the 12 -week combination treatment of glecaprevir and pibrentasvir for HCV GT4 
(a) 5' UTR-core region (655 nt)



(b) NS5B region (502 nt)

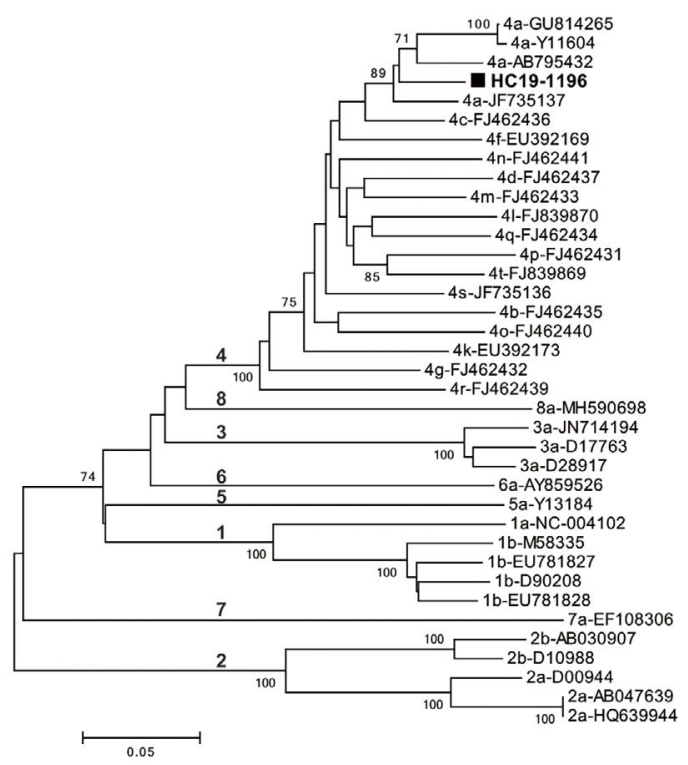

Figure 3. The phylogenetic trees constructed by the neighbor-joining method based on the hepatitis C virus (HCV)-5'-untranslated region (5'-UTR)-core region sequence (655 nt.) (a) and HCV-nonstructural protein (NS)5B region sequence (502 nt.) (b) of the $\mathrm{HCV}$ isolated from the present case (HC19-1196) as well as HCV strains of genotypes (GTs) 1-8. In addition to the isolated strain (HC191196/black square), 36 representative HCV strains are shown, including the HCV GT, subgenotype, and accession number. Bootstrap values $(\geqq 70 \%)$ are indicated for the nodes as a percentage of the data obtained from 1,000 resamplings. The scale bar is in units of nucleotide substitutions per site. The nucleotide sequences of the 5'-UTR-core region and NS5B of HC19-1196 are deposited as LC594551 and LC594552, respectively, in the DDBJ/GenBank databases.

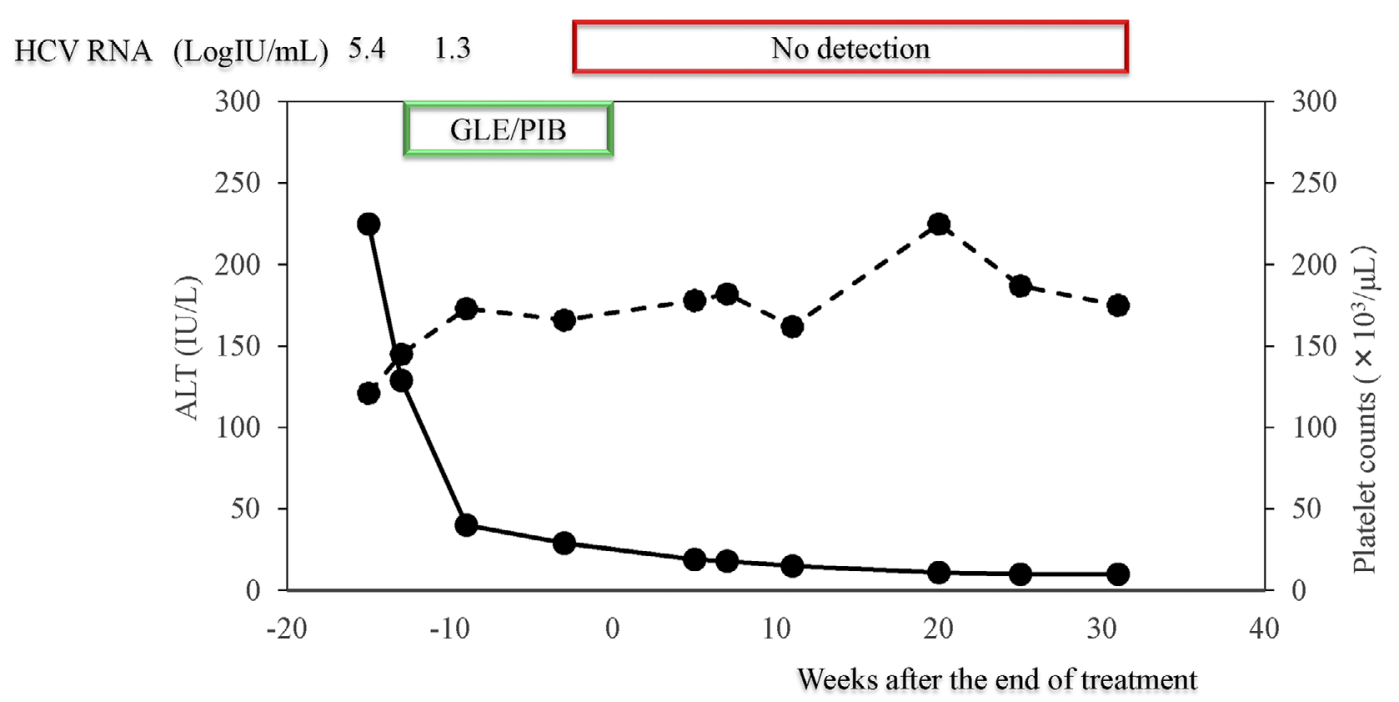

Figure 4. Clinical course of the present case. The combination of $300 \mathrm{mg}$ daily of glecaprevir (GLE) and $120 \mathrm{mg}$ daily of pibrentasvir (PIB) was given for 12 weeks. Solid line: ALT levels, Dotted line: Platelet counts.

in treatment-naïve patients with non-cirrhosis or cirrhosis led to $97.8 \%$ SVR rates (29) (Table 2). The ideal duration of this combination treatment should be further examined in the future.

Shiha et al. reported that the SVR rates after 12 and 24 weeks of $400 \mathrm{mg}$ daily sofosbuvir plus $60 \mathrm{mg}$ daily daclatasvir (HCV NS5A inhibitor), with or without 800$1,000 \mathrm{mg}$ daily ribavirin, were $96 \%$ and $93 \%$, respectively, in Egyptian patients with HCV GT4 (33). In combination treatment with $400 \mathrm{mg}$ daily sofosbuvir and $90 \mathrm{mg}$ daily le- 
Table 2. Sustained Virological Response Rates in HCV Genotype 4-infected Patients Treated with the Combination of Glecaprevir and Pibrentasvir.

\begin{tabular}{lcccc}
\hline Reference & Country & Type of diseases & $\begin{array}{c}\text { Treatment duration } \\
\text { (weeks) }\end{array}$ & $\begin{array}{c}\text { SVR12 rates } \\
\text { (SVR/Total patients) }\end{array}$ \\
\hline 22 & Italy & Non-LC, LC & $8-16$ & $100 \%(32 / 32)$ \\
23 & Israel & Non-LC, CC & 8 & $95.5 \%(63 / 66)$ \\
24 & USA & CC & 8 & $100 \%(2 / 2)$ \\
25 & USA & CC & 8 & $100 \%(13 / 13)$ \\
26 & UK & Non-LC, CC & $8-16$ & $99.4 \%(161 / 162)$ \\
27 & USA & Non-LC, LC (12-17 years) & 8 & $100 \%(3 / 3)$ \\
28 & Germany & Non-LC, LC & $8-12$ & $96.3 \%(26 / 27)$ \\
29 & Australia & Non-LC, LC & 12 & $97.8 \%(174 / 178)$ \\
30 & UK & Non-LC, CC & $8-16$ & $97.8 \%(178 / 182)$ \\
31 & Italy & Non-LC, LC & 8 & $100 \%(71 / 71)$ \\
32 & USA & Non-LC, CC & $8-12$ & $100 \%(175 / 175)$ \\
Our report & Japan & CC & 12 & $100 \%(1 / 1)$ \\
\hline
\end{tabular}

HCV: hepatitis C virus, SVR: sustained virological response, LC: liver cirrhosis, CC: compensated cirrhosis

dipasvir (HCV NS5A inhibitor), the SVR rates were 98\% and $94-98 \%$, respectively, in treatment-naïve and treatmentexperienced patients with HCV GT4 (16). Abd-Elsalam et al. reported that the SVR rate of the 24-week combination treatment of sofosbuvir and ribavirin was only $71.2 \%$ in 2,400 Egyptian patients with HCV GT4 infection and cirrhosis (34), and Ahmed et al. reported that the SVR rate of the 12-week combination treatment of sofosbuvir and velpatasvir (HCV NS5A inhibitor) was $99.6 \%$ in $120 \mathrm{HCV}$ GT4-infected patients (35).

The SVR rates of DAAs are known to be affected by several factors, such as the existence of HCV GTs, cirrhosis and/or HCC, and IL28B genotype (36-38). HCV NS5A resistance-associated substitutions (RASs), such as P32 deletion and R30E/Q54H/A92K, have effects on the treatment efficacy in patients with HCV GT1b treated with the combination of glecaprevir and pibrentasvir (39). Further studies will be needed to confirm the influence of these factors.

The present case was a treatment-naïve patient; however, an increased prevalence of RASs in treatment-experienced patients with HCV GT4 has been observed (40). Close attention should be paid to these situations in the treatment of HCV GT4-infected patients.

In Japan, 24-week combination treatment of sofosbuvir and ribavirin or 8-week combination treatment of glecaprevir and pibrentasvir is available for patients with HCV GT4 infection without cirrhosis. Patients with HCV GT4 infection and compensated cirrhosis can be treated with the 24week combination of sofosbuvir and ribavirin or the 12week combination of glecaprevir and pibrentasvir. We also can use the 12-week combination of sofosbuvir and velpatasvir for patients with decompensated cirrhosis. It is better for patients with HCV GT4 infection and non-cirrhosis to be treated with the 8-week combination of glecaprevir and pibrentasvir than the 12-week combination. At present, the American Association for the Study of Liver diseases (AASLD) recommends the 8-week combination treatment of glecaprevir and pibrentasvir for treatment-naïve patients with
HCV GT4 infection and compensated cirrhosis (41).

In conclusion, we encountered a Japanese case of HCV GT4 infection and cirrhosis that was successfully treated with the 12-week combination of glecaprevir and pibrentasvir. To our knowledge, this is the first documentation of the 12-week combination of glecaprevir and pibrentasvir for an HCV GT4-infected Japanese patient.

Author's disclosure of potential Conflicts of Interest (COI). Mitsuhiko Moriyama: Research funding, Towa Pharmaceutical.

\section{References}

1. Tateishi R, Uchino K, Fujiwara N, et al. A nationwide survey on non-B, non-C hepatocellular carcinoma in Japan: 2011-2015 update. J Gastroenterol 54: 367-376, 2019.

2. Kanda T, Lau GKK, Wei L, et al. APASL HCV guidelines of virus-eradicated patients by DAA on how to monitor HCC occurrence and HBV reactivation. Hepatol Int 13: 649-661, 2019.

3. Reddy KR, Pol S, Thuluvath PJ, et al. Long-term follow-up of clinical trial patients treated for chronic HCV infection with daclatasvir-based regimens. Liver Int 38: 821-833, 2018.

4. Simmonds P, Bukh J, Combet C, et al. Consensus proposals for a unified system of nomenclature of hepatitis $\mathrm{C}$ virus genotypes. Hepatology 42: 962-973, 2005.

5. Murphy DG, Sablon E, Chamberland J, et al. Hepatitis C virus genotype 7, a new genotype originating from central Africa. J Clin Microbiol 53: 967-972, 2015.

6. Borgia SM, Hedskog C, Parhy B, et al. Identification of a novel hepatitis $\mathrm{C}$ Virus genotype from punjab, india: expanding classification of hepatitis $\mathrm{C}$ virus into 8 genotypes. J Infect Dis 218: 1722-1729, 2018.

7. Omata M, Kanda T, Yokosuka O, et al. Features of hepatitis C virus infection, current therapies and ongoing clinical trials in ten Asian Pacific countries. Hepatol Int 9: 486-507, 2015.

8. Hayashi K, Fukuda Y, Nakano I, et al. Prevalence and characterization of hepatitis $\mathrm{C}$ virus genotype 4 in Japanese hepatitis $\mathrm{C}$ carriers. Hepatol Res 25: 409-414, 2003.

9. Omata M, Nishiguchi S, Ueno Y, et al. Sofosbuvir plus ribavirin in Japanese patients with chronic genotype $2 \mathrm{HCV}$ infection: An open-label, phase 3 trial. J Viral Hepat 21: 762-768, 2014.

10. Mizokami M, Yokosuka O, Takehara T, et al. Ledipasvir and sofosbuvir fixed-dose combination with and without ribavirin for 12 weeks in treatment-naive and previously treated Japanese patients 
with genotype 1 hepatitis $\mathrm{C}$ : an open-label, randomised, phase 3 trial. Lancet Infect Dis 15: 645-653, 2015.

11. Takehara T, Sakamoto N, Nishiguchi S, et al. Efficacy and safety of sofosbuvir-velpatasvir with or without ribavirin in HCV-infected Japanese patients with decompensated cirrhosis: an open-label phase 3 trial. J Gastroenterol 54: 87-95, 2019.

12. Toyoda H, Chayama K, Suzuki F, et al. Efficacy and safety of glecaprevir/pibrentasvir in Japanese patients with chronic genotype 2 hepatitis C virus infection. Hepatology 67: 505-513, 2018.

13. Chayama K, Suzuki F, Karino Y, et al. Efficacy and safety of glecaprevir/pibrentasvir in Japanese patients with chronic genotype 1 hepatitis $\mathrm{C}$ virus infection with and without cirrhosis. J Gastroenterol 53: 557-565, 2018.

14. Kumada H, Watanabe T, Suzuki F, et al. Efficacy and safety of glecaprevir/pibrentasvir in $\mathrm{HCV}$-infected Japanese patients with prior DAA experience, severe renal impairment, or genotype 3 infection. J Gastroenterol 53: 566-575, 2018.

15. Kumada H, Suzuki Y, Karino Y, et al. The combination of elbasvir and grazoprevir for the treatment of chronic $\mathrm{HCV}$ infection in Japanese patients: a randomized phase II/III study. J Gastroenterol 52: 520-533, 2017.

16. Kanda T, Matsuoka S, Moriyama M. Hepatitis C virus genotype 4-infection and interferon-free treatment in Egypt. Hepatol Int 12 291-293, 2018.

17. Aikawa T, Tsuda F, Ueno C, et al. Comparison of test results of serogrouping and core region PCR-based genotyping in patients with chronic hepatitis $\mathrm{C}$ virus infection: analysis of inderminate or discrepant cases and identification of a $2 b / 1 b$ recombinant HCV. Kanzo 57: 447-456, 2016 (in Japanese, Abstract in English).

18. Guerra J, Garenne M, Mohamed MK, et al. HCV burden of infection in Egypt: results from a nationwide survey. J Viral Hepat 19: 560-567, 2012

19. Choo QL, Kuo G, Weiner AJ, et al. Isolation of a cDNA clone derived from a blood-borne non-A, non-B viral hepatitis genome. Science 244: 359-362, 1989.

20. Kuo G, Choo QL, Alter HJ, et al. An assay for circulating antibodies to a major etiologic virus of human non-A, non-B hepatitis. Science 244: 362-364, 1989.

21. Forns X, Lee SS, Valdes J, et al. Glecaprevir plus pibrentasvir for chronic hepatitis $\mathrm{C}$ virus genotype $1,2,4,5$, or 6 infection in adults with compensated cirrhosis (EXPEDITION-1): a single-arm, open-label, multicentre phase 3 trial. Lancet Infect Dis 17: 10621068, 2017.

22. Aghemo A, Alberti A, Andreone $P$, et al. Effectiveness and safety of glecaprevir/pibrentasvir in chronic hepatitis $\mathrm{C}$ patients: Results of the Italian cohort of a post-marketing observational study. Dig Liver Dis S1590-8658: 30401-1.

23. Zuckerman E, Gutierrez JA, Dylla DE, et al. Eight weeks of treatment with glecaprevir/pibrentasvir is safe and efficacious in an integrated analysis of treatment-naïve patients with hepatitis $\mathrm{C}$ virus infection. Clin Gastroenterol Hepatol 18: 2544-2553.e6, 2020.

24. Flamm SL, Kort J, Marx SE, et al. Effectiveness of 8-week glecaprevir/pibrentasvir for treatment-naïve, compensated cirrhotic patients with chronic hepatitis C infection. Adv Ther 37: 2267-2274, 2020.

25. Brown RS Jr, Buti M, Rodrigues L, et al. Glecaprevir/pibrentasvir for 8 weeks in treatment-naïve patients with chronic HCV genotypes 1-6 and compensated cirrhosis: The EXPEDITION-8 trial. J Hepatol 72: 441-449, 2020.

26. Brown A, Welzel TM, Conway B, et al. Adherence to pangenotypic glecaprevir/pibrentasvir and efficacy in HCV-infected patients: a pooled analysis of clinical trials. Liver Int 40: 778-786, 2020.

27. Jonas MM, Squires RH, Rhee SM, et al. Pharmacokinetics, safety, and efficacy of glecaprevir/pibrentasvir in adolescents with chronic hepatitis C virus: Part 1 of the DORA Study. Hepatology 71: 456462, 2020.

28. Berg T, Naumann U, Stoehr A, et al. Real-world effectiveness and safety of glecaprevir/pibrentasvir for the treatment of chronic hepatitis C infection: data from the German Hepatitis C-Registry. Aliment Pharmacol Ther 49: 1052-1059, 2019.

29. Grebely J, Dore GJ, Alami NN, et al. Safety and efficacy of glecaprevir/pibrentasvir in patients with chronic hepatitis $\mathrm{C}$ genotypes 1-6 receiving opioid substitution therapy. Int J Drug Policy 66: 7379, 2019

30. Foster GR, Asselah T, Kopecky-Bromberg S, et al. Safety and efficacy of glecaprevir/pibrentasvir for the treatment of chronic hepatitis C in patients aged 65 years or older. PLoS One 14: e0208506, 2019.

31. D'Ambrosio R, Pasulo L, Puoti M, et al. Real-world effectiveness and safety of glecaprevir/pibrentasvir in 723 patients with chronic hepatitis C. J Hepatol 70: 379-387, 2019.

32. Krishnan P, Pilot-Matias T, Schnell G, et al. Pooled resistance analysis in patients with hepatitis $\mathrm{C}$ virus genotype 1 to 6 infection treated with glecaprevir-pibrentasvir in phase 2 and 3 clinical trials. Antimicrob Agents Chemother 62: e01249-18, 2018.

33. Shiha G, Soliman R, ElBasiony M, et al. Sofosbuvir plus daclatasvir with or without ribavirin for treatment of chronic HCV genotype 4 patients: real-life experience. Hepatol Int 12: 339-347, 2018.

34. Abd-Elsalam S, Sharaf-Eldin M, Soliman S, et al. Efficacy and safety of sofosbuvir plus ribavirin for treatment of cirrhotic patients with genotype 4 hepatitis $\mathrm{C}$ virus in real-life clinical practice. Arch Virol 163: 51-56, 2018.

35. Ahmed H, Abushouk AI, Attia A, et al. Safety and efficacy of sofosbuvir plus velpatasvir with or without ribavirin for chronic hepatitis $\mathrm{C}$ virus infection: a systematic review and meta-analysis. J Infect Public Health 11: 156-164, 2018.

36. Flamm S, Mutimer D, Asatryan A, et al. Glecaprevir/pibrentasvir in patients with chronic HCV genotype 3 infection: an integrated phase 2/3 analysis. J Viral Hepat 26: 337-349, 2019.

37. Sugiura A, Joshita S, Umemura T, et al. Past history of hepatocellular carcinoma is an independent risk factor of treatment failure in patients with chronic hepatitis $\mathrm{C}$ virus infection receiving direct-acting antivirals. J Viral Hepat 25: 1462-1471, 2018.

38. Mawatari S, Oda K, Kumagai $\mathrm{K}$, et al. Viral and host factors are associated with retreatment failure in hepatitis $\mathrm{C}$ patients receiving all-oral direct antiviral therapy. Hepatol Res 50: 453-465, 2020.

39. Sano T, Akuta N, Suzuki F, et al. Role of NS5A-L31/Y93 double wild-type in failure of glecaprevir/pibrentasvir double therapy in two patients with a history of direct-acting antiviral agent failure: an ultra-deep sequencing analysis. Intern Med 58: 2657-2662, 2019.

40. Merli M, Rossotti R, Travi G, et al. Sustained virological response with 16-week glecaprevir/pibrentasvir after failure to sofosbuvir/ velpatasvir in post-transplant severe $\mathrm{HCV}$ recurrence in HIV. Transpl Infect Dis 21: e13165, 2019.

41. The American Association for the Study of Liver Diseases and the Infectious Diseases Society of America. HCV Guidance: Recommendations for testing, managing, and treating hepatitis C. Simplified HCV treatment algorithm for treatment-naive adults with compensated cirrhosis [Internet]. [cited 2020 Dec 3]. Available from: https://www.hcvguidelines.org/treatment-naive/simplified-trea tment-compensated-cirrhosis

The Internal Medicine is an Open Access journal distributed under the Creative Commons Attribution-NonCommercial-NoDerivatives 4.0 International License. To view the details of this license, please visit (https://creativecommons.org/licenses/ by-nc-nd/4.0/).

(C) 2021 The Japanese Society of Internal Medicine

Intern Med 60: 2061-2066, 2021 